María Paola Podestá, María Cristina Hernández y Beatriz E. Bedoya Velásquez

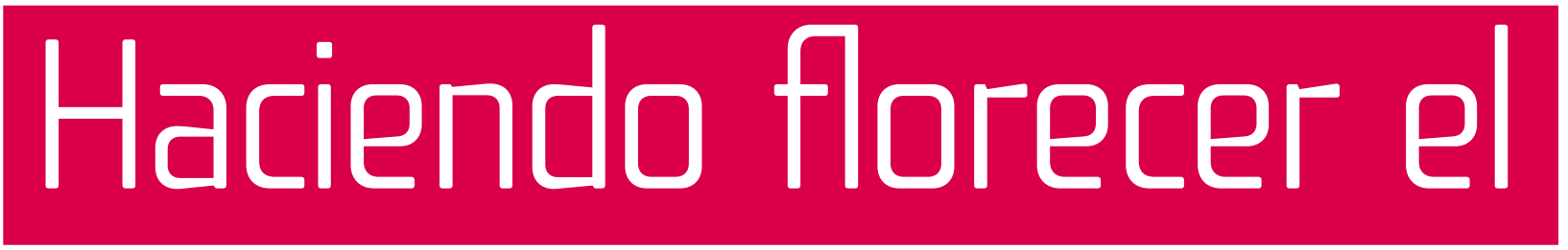

\title{
ecosistema. Análisis
}
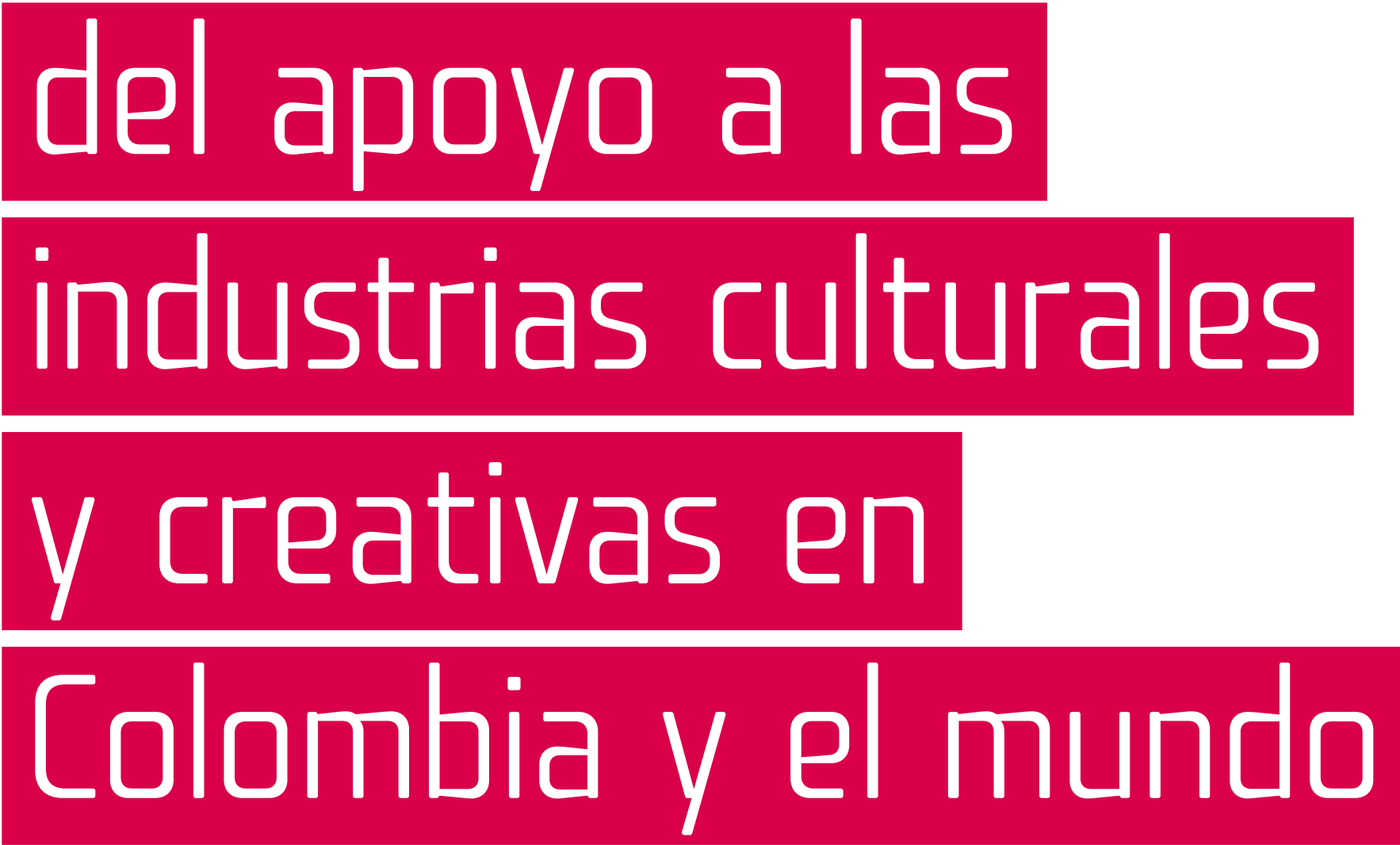


\section{Haciendo florecer el ecosistema.}

\section{Análisis del apoyo a las industrias}

\section{culturales y creativas en Colombia}

\section{y el mundo}

María Paola Podestá (0000-0001-7233-8009)

María Cristina Hernández (0000-0003-4311-190X)²

Beatriz E. Bedoya Velásquez (0000-0001-7626-9995) ${ }^{3}$

1 Jefe del Departamento de Negocios Internacionales de la Universidad EAFIT. mpodesta@eafit.edu.co

2 Docente de tiempo completo en la Universidad EAFIT en el Departamento de Ingeniería de Diseño. mhernand@eafit.edu.co

${ }^{3}$ Docente de tiempo completo de la Universidad EAFIT en el Departamento de Negocios Internacionales. Docente, investigadora y consultora en las áreas de economía creativa, emprendimiento, innovación, negocios internacionales e interculturalidad, y turismo. bbedoya1@eafit.edu.co

Fecha de recepción: 01 de octubre de 2019 | Fecha de aceptación: 29 de mayo de 2020

\section{Resumen}

La importancia de la Economía Creativa (EC) y de las Industrias Culturales y Creativas (ICC) hoy en día no puede ser subestimada. Esto ha llevado al surgimiento de ecosistemas de emprendimiento cultural y creativo que configuran una oferta de servicios de formación y apoyo para fortalecer el sector. Este artículo presenta resultados de una investigación sobre el estado de dicha oferta en el mundo, Iberoamérica y Colombia. Para el mundo e Iberoamérica se revisaron en línea 238 instituciones. Para el contexto colombiano, la información se recopiló mediante entrevistas estructuradas a 25 representantes de diferentes organismos. El artículo presenta un mapeo de organismos constituyentes de ecosistemas y un inventario de la oferta de este tipo de servicios identificados. Los hallazgos presentados permiten identificar oportunidades de crecimiento y fortalecimiento para el emprendimiento cultural y creativo en el mundo, y señala aspectos críticos para el ámbito colombiano, en relación con referentes internacionales.

Palabras clave | Ecosistema, Emprendimiento, Industrias Culturales y Creativas, Economía Creativa 


\title{
Making the Ecosystem Bloom. Exploring the Supports for the Cultural and Creative Industries in Colombia and the World
}

\begin{abstract}
The importance of the Creative Economy (CE) and of the Cultural and Creative Industries $(\mathrm{CCl})$ today cannot be underestimated. This has led to the emergence of cultural and creative entrepreneurship ecosystems that configure an offer of training and support services for strengthening the sector. This article presents results of a research on the status of said offer in the world, Latin America and Colombia. For the world and Latin America, 238 institutions were reviewed online. For the Colombian context, the information was collected through structured interviews with 25 representatives of different organizations. The article presents a mapping of organisms and ecosystems and an inventory of the offer of this type of services. The findings presented allow the identification of opportunities for growth and strengthening for cultural and creative entrepreneurship in the world, and points out critical aspects for the Colombian environment, in relation to international references.
\end{abstract}

Keywords | Ecosystem, Entrepreneurship, Cultural and Creative Industries, Creative Economy

\section{Copyright}

Centro de Diseño y Comunicación, S.C.C 2020. Este es un artículo de acceso abierto distribuido según los términos de la Licencia de Atribución de Creative Commons (CC BY-NC-ND 4.0), que permite la descarga, el uso y la distribución en cualquier medio, sin propósitos comerciales y sin derivadas, siempre que se acredite al autor original y la fuente. 


\section{Introducción}

La Economía Creativa (EC) se refiere a la gama de actividades económicas relacionadas con la generación y explotación de la creatividad y la propiedad intelectual, las cuales dan origen a las ICC (UNCTAD, 2013), y es un sector cuya importancia no debe ser subestimada. La Organización de las Naciones Unidas (ONU), bajo el organismo estructural de la Conferencia de las Naciones Unidas sobre Comercio y Desarrollo (UNCTAD), ha identificado a la EC y a las ICC como categorías de gran relevancia en términos de desarrollo, crecimiento y comercio internacional; el tema ha llegado a ocupar un lugar predominante en agendas de desarrollo de diversos países, y se ha consolidado como un tema importante en sistemas políticos y educativos de todo el mundo (Bedoya, 2016). La UNCTAD rastrea en más de 126 países y regiones un sinnúmero de estrategias para la promoción y el fortalecimiento de los diferentes sectores que hacen parte de las ICC (UNCTAD, 2008; UNCTAD, 2010; UNCTAD, 2013), todas ellas con el objetivo de propiciar la formación empresarial y el desarrollo de actividades empresariales regionales asociadas a la EC (Mack y Mayer, 2016). Para la sostenibilidad de las ICC es necesario que haya un trabajo en equipo; que en cada sociedad sean posibles las ideas de los individuos, asunto que, a pesar de parecer contradictorio, es esencial, y se da en la medida en que la sociedad las acepte y reconozca dentro de sus valores, de manera que las prácticas de las mismas se expandan y repliquen en cada sociedad.

La identificación de ecosistemas para el emprendimiento ha sido de interés para múltiples académicos (Cohen, 2006; Feld, 2012; Isenberg, 2010; Mason y Brown, 2014; Mack y Mayer, 2016). Si bien identificar los ecosistemas tiene una relevancia, este es apenas el primer paso para comprender e intervenir un sector que está en consolidación en el mundo y en Colombia. Es necesario establecer las relaciones que se dan entre los componentes de dicho ecosistema, más específicamente, las relaciones empresariales entre Industrias Creativas y Culturales (ICC), a fin de lograr una participación más pertinente, ajustada a las realidades de lo que existe y de lo que es posible o necesario crear u ofrecer. 
El propósito de este trabajo consiste en explorar y conocer la oferta de servicios de formación y apoyo para el sector de la ICC en Colombia, con el fin de identificar los actores y las relaciones entre ellos. En congruencia con el enfoque propuesto para realizar la exploración, se asumió, en un sentido metafórico, la noción de ecosistema propuesta por Capra (1997), concebido como una comunidad sostenible que responde a los siguientes principios.

- Interdependencia: se refiere a las relaciones de dependencia necesarias para el funcionamiento de los miembros de un ecosistema. Estas relaciones no son necesariamente de causa-efecto.

- Naturaleza cíclica: se refiere a los procesos ecológicos, es decir, a la transformación de insumos en desechos que son, a su vez, insumo para otros miembros del ecosistema.

- Asociación: se refiere a la tendencia a cooperar, asociarse y establecer vínculos.

- Flexibilidad: se refiere a la existencia de fluctuaciones constantes típicas de un sistema y a la capacidad del mismo para adaptarse a dichas fluctuaciones a través de la cantidad de conexiones entre los miembros del ecosistema.

- Sostenibilidad: capacidad del sistema para mantenerse en el tiempo, directamente relacionado con la flexibilidad.

Para realizar el estudio se realizó un rastreo en línea de organismos en el mundo que ofrecen formación y apoyo, mediante una revisión documental, la cual sentó las bases para la posterior categorización e identificación de veinticinco organismos públicos y privados en Colombia que realizan las mismas acciones. Los resultados obtenidos fueron la base para caracterizar los ecosistemas de apoyo al emprendimiento cultural y creativo, teniendo en cuenta los servicios que se ofrecen a las ICC.

En relación con el alcance de este estudio, la investigación no exploró los aspectos relacionados con la naturaleza cíclica y la sostenibilidad de los ecosistemas, ya que no pretendió identificar o evaluar los resultados de los servicios de formación y apoyo para el emprendimiento cultural y creativo; sin embargo, los aspectos 
de interdependencia, asociación y flexibilidad sí fueron considerados. En consistencia con este marco conceptual, se refiere a las instituciones como organismos.

La investigación que se realizó es de orden cualitativo, descriptivo y exploratorio. El estudio se aproxima inductivamente a la construcción del conocimiento sobre la formación y el apoyo al emprendimiento cultural y creativo, partiendo de una visión internacional que se concreta en un análisis más detallado a nivel local para el caso de Colombia. Los servicios de formación y apoyo se comprendieron como las manifestaciones de las interacciones ecosistémicas que articulan a empresarios y organismos de promoción. Las fuentes de información que sirvieron de base para este estudio fueron las páginas de los organismos que conforman el universo, así como entrevistas estructuradas a representantes que ofrecen formación y apoyo a las ICC en Colombia.

\section{Ámbito internacional}

El primer componente se desarrolló mediante una investigación documental en línea para la recopilación de las iniciativas internacionales que apoyan el emprendimiento cultural y creativo. La búsqueda se realizó en inglés y en español, lo cual supone una limitación de los resultados aquí presentados a aquellos que cuenten con indexación en estos idiomas en el motor de búsqueda Google. Es importante aclarar que, si bien Google puede no ser la indexación más completa y rigurosa para el rastreo, se asume como el punto de partida de base mediante el cual empresarios del sector se aproximaron a la búsqueda de servicios de formación y apoyo. Se implementaron criterios de saturación para determinar el alcance de la exploración en los resultados, excediendo lo que sería una búsqueda regular en el motor. De igual forma, se determinó un criterio de validez y relevancia de los resultados en el buscador, considerando solamente ofertas encontradas hasta las diez primeras páginas de resultados de cada búsqueda, puesto que, en promedio, a partir de esa página, los resultados empezaron a mostrar desactualizaciones considerables, información desvinculada a los criterios de búsqueda y vínculos rotos o sin contenido. 
Se construyó una matriz en la que se identifican las categorías para cada institución revisada (Tabla 1). Cabe resaltar que, en varios casos, una misma institución ofrece varios tipos de iniciativas que apoyan el desarrollo del emprendimiento cultural y creativo.

Tabla 1. Información general sobre instituciones e iniciativas

\begin{tabular}{l}
\multicolumn{1}{c}{ País } \\
\hline Institución \\
\hline Sistema de apoyo \\
\hline Programas o iniciativas \\
\hline Página web \\
\hline Áreas de trabajo \\
\hline Instituciones asociadas \\
\hline
\end{tabular}

La categoría "país" permitió identificar los países en los cuales el apoyo al emprendimiento cultural y creativo se encuentra muy desarrollado y consolidado a través de múltiples y diversos tipos de instituciones, tanto públicas como privadas. Esta información permitió contar con datos adicionales que se utilizaron al momento de realizar las entrevistas con las instituciones de promoción revisadas en el posterior trabajo de campo en Colombia.

La categoría "sistema de apoyo" permitió hacer una clasificación de los tipos de servicios que proveen las diferentes instituciones. A partir de la información arrojada por esta categoría fue posible clasificar las instituciones en dos grupos, aquellas que prestan servicios de formación (técnica, profesional, complementaria y/o vocacional), y aquellas que prestan servicios de apoyo (consultoría, financiación, investigación, eventos, incubación, información y otros similares). Estas clasificaciones, sin embargo, no son excluyentes, queriendo decir con esto que algunas instituciones pueden prestar ambos tipos de servicios. 
La identificación de las áreas o temáticas de trabajo en cada institución permitió establecer los temas en los cuales se están concentrando los esfuerzos, tanto en formación, como en otros servicios de apoyo. Se identificó una oferta de servicios de formación en las siguientes temáticas:

- Prácticas en oficios/talentos: formación en carreras/programas específicos de corte creativo/cultural (música, teatro, danza, escritura, etc.).

- Gestión empresarial: formación en saberes puntuales relacionados con la administración y la dirección de ICC.

- Investigación: formación en la disciplina investigativa relevante para el contexto de la industria.

- Emprendimiento: formación en habilidades para el emprendimiento cultural y creativo.

- Desarrollo de políticas públicas: formación en perfiles profesionales para la participación en el desarrollo y creación de políticas públicas.

- Teoría y contexto: formación en los contenidos y conceptos base de la EC y de las ICC.

- Gestión comercial: formación en habilidades para la comercialización efectiva de bienes y servicios culturales y creativos.

- Actualización: formación para actualización a empresarios de corte más empírico en temas generales de gestión y EC.

- Formación para la sensibilidad: formación para desarrollar una conciencia de la industria, su categorización, su estado actual, sus prácticas y otros temas relevantes de la EC.

- Gestión cultural: formación de perfiles profesionales orientados al funcionamiento como gestores culturales para la industria.

De igual forma, se identificó la siguiente oferta de servicios de apoyo:

- Apoyo financiero: servicios de préstamo o acceso a fondos para la inversión.

- Oferta de información propia/otros sobre servicios para el sector: servicios de presentación de anuncios, oportunidades, capacitaciones, y otra información relevante para el sector, incluyendo estudios o mediciones. 
- Investigaciones del sector: servicios de desarrollo de investigaciones sobre la EC y las ICC.

- Promoción*: servicios varios de proyección de las ICC.

- Consultoría*: servicios de acompañamiento para el fortalecimiento de las ICC.

- Facilitación de Networking: servicios para facilitar la interconexión y asociatividad entre empresarios del sector.

- Incubación (apoyo en recursos no financieros): servicios de apoyo en las etapas de inicio de los proyectos empresariales.

- Creación de políticas públicas: servicios de representación y participación en escenarios de creación y decisión sobre políticas públicas.

Las áreas de promoción y consultoría se subdividen a su vez como se ilustra en la Tabla 2, haciendo referencia a ciertas actividades específicas que, en general, aluden al mismo esfuerzo.

\section{Tabla 2. Subclasificaciones: Promoción y Consultoría}

\begin{tabular}{ll} 
Actividad & \\
\hline $\begin{array}{l}\text { Promoción } \\
\text { (d) }\end{array}$ & $\begin{array}{l}\text { Consultoría } \\
(\mathrm{e})\end{array}$ \\
\hline (d1) Eventos & $(\mathrm{e} 1)$ Estudios de mercado \\
\hline (d2) Ruedas de negocios & $(\mathrm{e} 2)$ Diagnóstico \\
\hline (d3) Ferias & $(\mathrm{e} 3)$ Potenciar capacidades \\
\hline (d4) Muestras y exposiciones & \\
\hline (d5) Festivales & \\
\hline (d6) Misiones & Fuente: Elaboración propia.
\end{tabular}

Finalmente, la categoría "instituciones asociadas" permitió expandir la base de organismos que se iba construyendo e, igualmente, hizo evidente la existencia de un entramado interinstitucional. Esto se evidenció a partir de las descripciones encontradas en las que las mismas instituciones referencian otras entidades con quienes tienen alianzas para la prestación de los servicios o la oferta de información. Dicha observación se convirtió en el sustento que valida el uso de la noción 
de ecosistema para referirse al grupo de instituciones, sus relaciones, el propósito de las mismas y el conjunto de servicios que prestan.

De la revisión global fue posible identificar que las iniciativas y programas ofrecidos difieren entre los países de Latinoamérica y el resto del mundo. Aunque las temáticas se mantienen, la cantidad de esfuerzo dedicado varía de acuerdo a las diferentes condiciones de desarrollo en las que se encuentra el sector del emprendimiento cultural y creativo en países desarrollados, versus los países en vía de desarrollo. Esto se hizo evidente al observar las cantidades y los tipos de iniciativas en las cuales se concentran los esfuerzos de los países encontrados. Por esta razón, se hace la distinción entre instituciones del mundo y las iberoamericanas, incluyendo los programas de apoyo españoles a la comunidad latina en este segundo grupo, ya que se evidenciaron múltiples iniciativas en convenios con instituciones de este país (bajo la categoría Iberoamérica se excluye a Portugal, ya que no fue posible identificar ofertas puntuales de este país, como sí lo fueron las de España). Se utiliza estadística descriptiva para presentar regularidades encontradas a partir de la tabulación de la información anteriormente explicada.

\section{Ámbito local}

El segundo componente consistió en la realización del trabajo de campo con instituciones colombianas que ofrecen servicios de formación y apoyo para el desarrollo del emprendimiento cultural y creativo mediante entrevistas estructuradas. Para guardar relación con los tipos de organismos identificados y revisados en el ámbito internacional, la selección de los organismos a entrevistar en el ámbito local (Colombia) incluyó entidades de regulación y control nacionales y regionales, organismos regionales articuladores, y organismos locales que operan y ejecutan servicios de formación y apoyo. Con base en el sistema categorial construido para la clasificación de los servicios de formación y apoyo recopilados en el ámbito internacional, se elaboró un protocolo de preguntas estructurado para dar cuenta de los mismos campos de información en el trabajo presencial con los organismos del ámbito local. Los interrogantes fueron cerrados en su mayoría, 
con algunas preguntas abiertas para explorar posturas adicionales sobre la percepción frente a la oferta de los servicios. A pesar de las críticas hechas sobre la entrevista estructurada en cuanto a su formalidad (Vargas, 2012), la necesidad de poder comparar el ámbito local con el internacional, demandó precisamente el sondeo a través de las mismas categorías de análisis y clasificación, logrando, como lo indican del Rincón et al. (1995), explorar el número limitado de categorías relevantes al mapeo.

La identificación y selección de los organismos a entrevistar en Colombia se derivó, en gran medida, del rastreo realizado en la búsqueda de organismos internacionales, esta revisión bibliográfica del ámbito internacional aclaró el tipo de instituciones que deberían explorarse en el trabajo de campo en Colombia, incluyendo instituciones de regulación y control, e instituciones ejecutoras; la experiencia del equipo investigador en la industria, proveyó otros referentes a entrevistar que no se hicieron evidentes en los resultados indexados en el motor de búsqueda usado.

La Tabla 3 presenta información demográfica de los 25 organismos entrevistados. En algunas instituciones se entrevistaron varias personas que trabajan con la promoción del emprendimiento cultural y creativo desde diferentes áreas. Todas las personas entrevistadas pertenecen a los niveles directivos y de coordinación de sus respectivos organismos, teniendo a su cargo el diseño y la ejecución de programas de formación y/o apoyo para la ICC.

\section{Tabla 3. Instituciones para el trabajo de campo}

\begin{tabular}{|c|c|c|c|c|}
\hline Institución & Pública & Privada & Nivel & Entrevistas \\
\hline Bogotá & & & & 8 \\
\hline Prana & & $x$ & 3 & \\
\hline Ministerio de Cultura & $x$ & & 1 & \\
\hline Ministerio de Industria, Turismo y Comercio & $x$ & & 1 & \\
\hline Universidad Javeriana - Bogotá & & $x$ & 3 & \\
\hline
\end{tabular}




\begin{tabular}{|c|c|c|c|c|}
\hline $\mathrm{BID}$ & & $x$ & 1 & \\
\hline Universidad del Rosario & & $x$ & 3 & \\
\hline Cámara de Comercio de Bogotá & & $x$ & 2 & \\
\hline British Council & & $x$ & 1 & \\
\hline Cali & & & & 8 \\
\hline Comfandi & & $x$ & 3 & \\
\hline Alcaldía de Cali - Secretaría de Cultura & $x$ & & 2 & \\
\hline Universidad ICESI & & $x$ & 3 & \\
\hline Cámara de Comercio de Cali & & $x$ & 2 & \\
\hline Universidad del Valle & $x$ & & 3 & \\
\hline Cartagena & & & & 2 \\
\hline Mercado del Caribe & & $x$ & 3 & \\
\hline Cámara de Comercio de Cartagena & & $x$ & 2 & \\
\hline Manizales & & & & 3 \\
\hline Universidad Nacional- Manizales & $x$ & & 3 & \\
\hline Universidad de Caldas & $x$ & & 3 & \\
\hline Medellín & & & & 4 \\
\hline Alcaldía de Medellín & $x$ & & 2 & \\
\hline Comfama & & $x$ & 3 & \\
\hline Universidad de Antioquia & $x$ & & 3 & \\
\hline Asencultura & & $x$ & 3 & \\
\hline Total Entrevistas & & & & 25 \\
\hline
\end{tabular}

Fuente: Elaboración propia

\section{Hallazgos}

En total se revisaron 125 instituciones internacionales (esta cifra se compone de 57 instituciones de formación y 68 de apoyo), y 113 instituciones en Iberoamérica (esta cifra se compone de 35 instituciones de formación y 78 de apoyo, dentro de las cuales se identificaron un total de 81 iniciativas de apoyo, queriendo decir con esto, que algunas instituciones ofrecen múltiples servicios de apoyo). La Tabla 4 presenta el número de organismos identificados en el mundo que ofrecen servicios de apoyo organizados por país. La Tabla 5 presenta la misma información para el caso de los organismos que ofrecen servicios de formación. 
Tabla 4. Grupo de instituciones de apoyo

\begin{tabular}{|c|c|}
\hline País & No. Organismos \\
\hline Australia & 5 \\
\hline Canadá & 1 \\
\hline China & 2 \\
\hline Dinamarca & 5 \\
\hline Estados Unidos & 15 \\
\hline Finlandia & 2 \\
\hline India & 1 \\
\hline Islandia & 2 \\
\hline Noruega & 2 \\
\hline Reino Unido & 10 \\
\hline Rusia & 1 \\
\hline Suecia & 6 \\
\hline Unión Europea & 1 \\
\hline Argentina & 11 \\
\hline Bolivia & 3 \\
\hline Brasil & 7 \\
\hline Chile & 9 \\
\hline Colombia & 8 \\
\hline Costa Rica & 8 \\
\hline Ecuador & 7 \\
\hline España & 4 \\
\hline Guatemala & 4 \\
\hline Honduras & 5 \\
\hline México & 6 \\
\hline Paraguay & 2 \\
\hline $\begin{array}{l}\text { Instituciones } \\
\text { Internacionales }\end{array}$ & 19 \\
\hline
\end{tabular}

\section{Tabla 5. Grupo de instituciones} de formación

\begin{tabular}{lc} 
País & No. Organismos \\
\hline Australia & 2 \\
\hline Canadá & 1 \\
\hline China & 2 \\
\hline Dinamarca & 4 \\
\hline Estados Unidos & 17 \\
\hline Finlandia & 2 \\
\hline Irlanda & 1 \\
\hline Islandia & 1 \\
\hline Letonia & 1 \\
\hline Noruega & 3 \\
\hline Reino Unido & 18 \\
\hline Rusia & 1 \\
\hline Suecia & 3 \\
\hline Taiwán & 1 \\
\hline Argentina & 11 \\
\hline Brasil & 3 \\
\hline Colombia & 1 \\
\hline Costa Rica & 8 \\
\hline Ecuador & 1 \\
\hline España & 9 \\
\hline México & \\
\hline Fuente: elaboración propia & 10 \\
\hline
\end{tabular}

Una vez identificados los países para el estudio de iniciativas en Latinoamérica, se clasificaron éstas según fueran los organismos: públicos, privados, mixtos. Esta forma de clasificación de los organismos permitió identificar en dónde se concentran los recursos para la formación en temas relacionados con el emprendimiento cultural y creativo. 
Para los organismos de apoyo, adicional a la clasificación anterior, se identificó si los servicios prestados eran de alcance nacional o internacional, con el fin de observar las tendencias en la promoción y el desarrollo del emprendimiento cultural y creativo.

En términos generales se encontró que, de los 125 organismos internacionales revisados, 63 son públicos, 61 son privados y solo uno es mixto. Por su parte, de los 113 organismos iberoamericanos revisados 77 son públicos, y 39 son privados (la diferencia de tres sobre el total se explica debido a que la categoría pública/ privada se asignaban en referencia a los programas ofrecidos por los organismos y no tomando el organismo como punto de referencia, así, como se mencionó anteriormente, aunque se revisaron 78 organismos, en total se valoraron 81 iniciativas).

Los organismos se clasificaron según el área de formación a la que le apuntan y según el tipo de servicio de apoyo que prestan, como se muestra en el ejemplo de las Tablas 6 y 7.

Tabla 6. Clasificación de las instituciones de formación según el área en la que forman

\begin{tabular}{l|c|c} 
Área & Institución 1 & Institución 2 \\
\hline Prácticas (oficios y talentos) & $\mathrm{x}$ & \\
\hline Gestión empresarial & & $\mathrm{x}$ \\
\hline Investigación & $\mathrm{x}$ & \\
\hline Emprendimiento & & \\
\hline Desarrollo de políticas públicas & $\mathrm{x}$ & $\mathrm{x}$ \\
\hline Teoría y contexto & & $\mathrm{x}$ \\
\hline Gestión comercial & & $\mathrm{x}$ \\
\hline Actualización & & \\
\hline Formación para la sensibilidad & $\mathrm{x}$ & \\
\hline Gestión cultural & $\mathrm{x}$ & \\
\hline Total (\%) & $\%$ & \\
\hline
\end{tabular}


Tabla 7. Clasificación de las instituciones de apoyo según la actividad que realizan

\begin{tabular}{l|c|c} 
Área & Institución 1 & Institución 2 \\
\hline Financiación & $\mathrm{x}$ & $\mathrm{x}$ \\
\hline Información & & \\
\hline Investigación & & \\
\hline Promoción & $\mathrm{x}$ & $\mathrm{x}$ \\
\hline Eventos & & $\mathrm{x}$ \\
\hline Ruedas de negocios & & \\
\hline Ferias & & \\
\hline Muestras/Exposiciones & $\mathrm{x}$ & \\
\hline Festivales & & \\
\hline Misiones & $\mathrm{x}$ & \\
\hline Consultoría & & $\mathrm{x}$ \\
\hline Estudios de Mercado & & \\
\hline Diagnóstico & $\mathrm{x}$ & \\
\hline Potenciar capacidades & & \\
\hline Networking & & \\
\hline Incubación & & \\
\hline Creación de políticas públicas & & \\
\hline Total (\%) & & \\
\hline & & \\
\hline
\end{tabular}

Fuente: elaboración propia

De la información derivada de las tablas 6 y 7 se hacen dos lecturas: la primera hace referencia a la sumatoria horizontal del número de servicios de formación y apoyo, y el porcentaje correspondiente que cada organismo promueve del total de las áreas identificadas; la segunda permite observar el total de servicios ofrecidos por temática específica (sumatoria vertical). De esta última lectura resultan 4 gráficas que expresan los porcentajes de esfuerzos concentrados por áreas de formación y apoyo en el mundo e Iberoamérica (Figuras 1 - 4). 


\section{Porcentaje de esfuerzos concentrados por áreas de formación en el mundo}

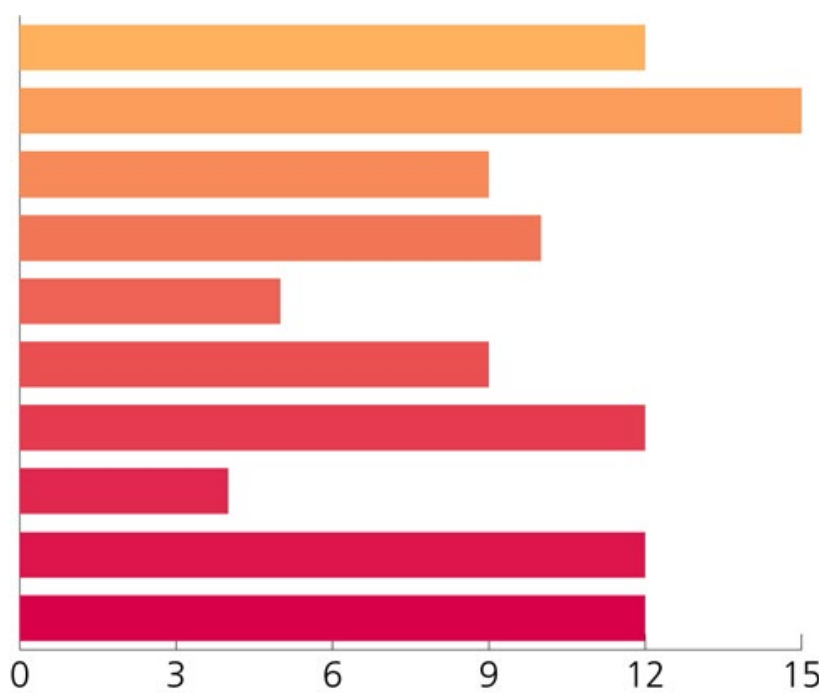

\section{Porcentaje de esfuerzos concentrados por áreas de apoyo en el mundo}

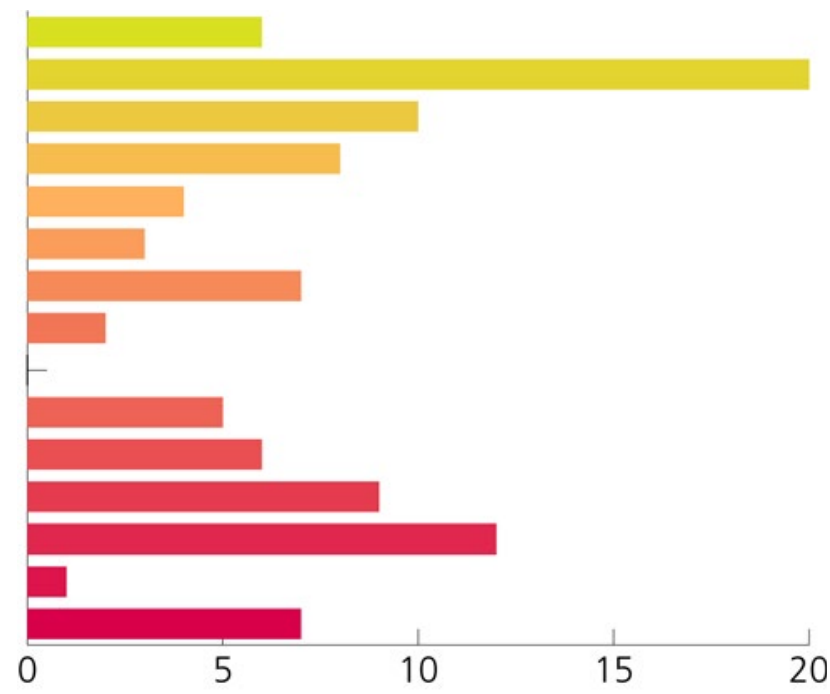

$12 \%$ Prácticas $=$ oficios y talentos

15\% Gestión empresarial

9\% Investigación

$10 \%$ Emprendimiento

$\mathbf{5 \%} \quad$ Desarrollo de políticas públicas

$\mathbf{9 \%}$ Teoría y contexto

$12 \%$ Gestión comercial

4\% Actualización

12\% Formación para la sensibilidad

12\% Gestión cultural
Figura 1

Porcentaje de esfuerzos concentrados por áreas de formación en el mundo. Fuente: Elaboración propia.
6\% Financiación

$\mathbf{2 0 \%}$ Información

$10 \%$ Investigación

$\mathbf{8 \%} \quad$ Promoción de eventos

4\% Promoción Ruedas de negocios

3\% Promoción Ferias

7\% Promoción Muestras/Exposiciones

$\mathbf{2 \%} \quad$ Promoción Festivales

0\% Promoción Misiones

$\mathbf{5 \%} \quad$ Consultoría Estudios mercado

$\mathbf{6 \%}$ Consultoría Diagnóstico

9\% Consultoría Potenciar capacidades

$12 \%$ Networking

$1 \%$ Incubación

7\% Creación de políticas públicas
Figura 2

Porcentaje de esfuerzos concentrados por áreas de apoyo en el mundo.

Fuente: Elaboración propia. 


\section{Porcentaje de esfuerzos concentrados por áreas de formación en Iberoamérica}

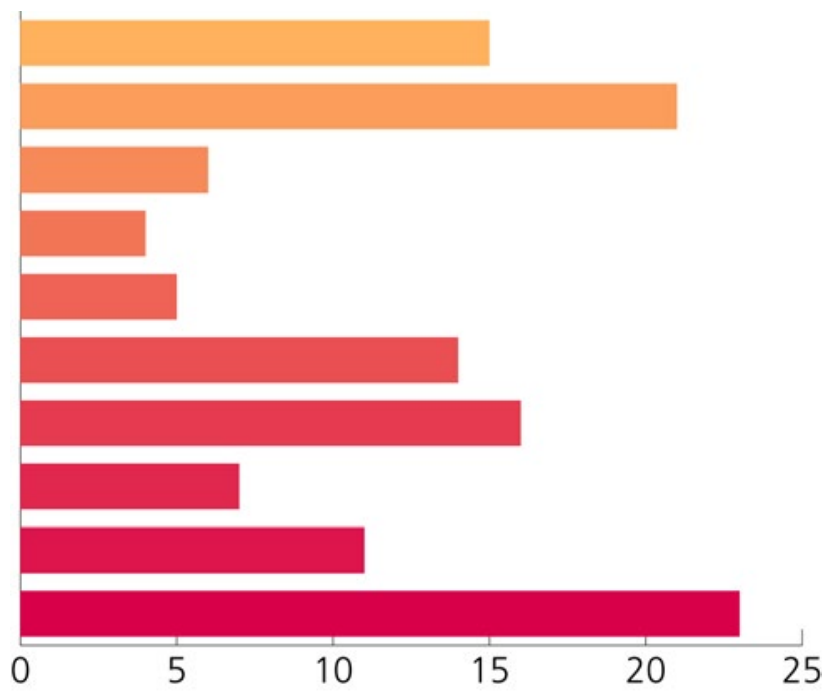

15\% Prácticas $=$ oficios y talentos

$\mathbf{2 1 \%}$ Gestión empresarial

6\% Investigación

$\mathbf{4 \%}$ Emprendimiento

$\mathbf{5 \%} \quad$ Desarrollo de políticas públicas

$\mathbf{1 4 \%}$ Teoría y contexto

$16 \%$ Gestión comercial

7\% Actualización

11\% Formación para la sensibilidad

23\% Gestión cultural
Figura 3

Porcentaje de esfuerzos concentrados por áreas de formación en Iberoamérica. Fuente: Elaboración propia.

\section{Porcentaje de esfuerzos concentrados por áreas de apoyo en Iberoamérica}

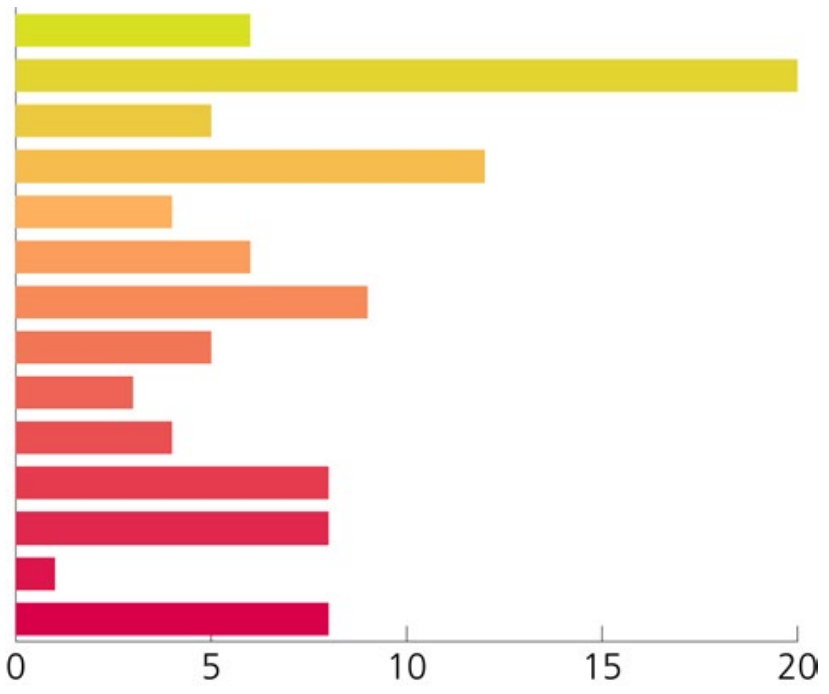

6\% Financiación

$\mathbf{2 0 \%}$ Información

$\mathbf{5 \%}$ Investigación

12\% Promoción de eventos

4\% Promoción Ruedas de negocios

6\% Promoción Ferias

$\mathbf{9 \%} \quad$ Promoción Muestras/Exposiciones

$\mathbf{5 \%}$ Promoción Festivales

0\% Promoción Misiones

3\% Consultoría Estudios mercado

4\% Consultoría Diagnóstico

$\mathbf{8 \%}$ Consultoría Potenciar capacidades

$\mathbf{8 \%} \quad$ Networking

$1 \%$ Incubación

$\mathbf{8 \%} \quad$ Creación de políticas públicas
Figura 4

Porcentaje de esfuerzos concentrados por áreas de apoyo en el Iberoamérica. Fuente: Elaboración propia. 
Adicionalmente, se realizó un análisis de referencias cruzadas entre las diferentes categorías de clasificación así:

- Porcentaje de instituciones públicas y privadas del total revisado.

- Porcentaje de instituciones que operan nacional o internacionalmente.

- Porcentaje de instituciones que ofrecen un servicio de apoyo específico.

- Porcentaje de instituciones que forman en una temática específica.

La Tabla 8 presenta los valores que resultan de estos análisis.

Tabla 8. Síntesis servicios de formación y apoyo en Iberoamérica y el mundo

Iberoamérica

\begin{tabular}{|c|c|c|c|c|}
\hline & & & \multicolumn{2}{|c|}{ Alcance } \\
\hline & & & \multirow{2}{*}{$\begin{array}{c}\text { Nacio- } \\
\text { nal } \\
(79 \%)\end{array}$} & \multirow{2}{*}{$\begin{array}{c}\text { Interna- } \\
\text { cional } \\
(21 \%)\end{array}$} \\
\hline & Pública & Privada & & \\
\hline Servicios de Formacióna & $80 \%$ & $20 \%$ & - & - \\
\hline Prácticas y Oficios & $37 \%$ & $11 \%$ & - & - \\
\hline Gestión Empresarial & $51 \%$ & $14 \%$ & - & - \\
\hline Investigación & $20 \%$ & $0 \%$ & - & - \\
\hline Emprendimiento & $9 \%$ & $3 \%$ & - & - \\
\hline Políticas Públicas & $11 \%$ & $6 \%$ & - & - \\
\hline Teoría y Contexto & $51 \%$ & $51 \%$ & - & - \\
\hline Gestión Comercial & $34 \%$ & $34 \%$ & - & - \\
\hline Actualización & $11 \%$ & $11 \%$ & - & - \\
\hline Sensibilidad & $29 \%$ & $6 \%$ & - & - \\
\hline Gestión Cultural & $60 \%$ & $11 \%$ & - & - \\
\hline Servicios de Apoyo & $80 \%$ & $20 \%$ & - & - \\
\hline Financiación ${ }^{b}$ & $21 \%$ & $2 \%$ & $20 \%$ & $4 \%$ \\
\hline Oferta de información & $44 \%$ & $32 \%$ & $60 \%$ & $16 \%$ \\
\hline Investigaciones del sector & $14 \%$ & $5 \%$ & $15 \%$ & $4 \%$ \\
\hline Promoción Eventos & $25 \%$ & $17 \%$ & $33 \%$ & $12 \%$ \\
\hline Ruedas de negocios & $6 \%$ & $9 \%$ & $10 \%$ & $5 \%$ \\
\hline Ferias & $14 \%$ & $10 \%$ & $17 \%$ & $5 \%$ \\
\hline Muestras y Expo & $19 \%$ & $16 \%$ & $23 \%$ & $10 \%$ \\
\hline Festivales & $7 \%$ & $10 \%$ & $11 \%$ & $6 \%$ \\
\hline
\end{tabular}

Mundo

\begin{tabular}{|c|c|c|c|c|}
\hline \multirow[b]{3}{*}{ Pública } & \multirow[b]{3}{*}{ Privada } & \multicolumn{3}{|c|}{ Alcance } \\
\hline & & \multirow{2}{*}{$\begin{array}{c}\text { Nacio- } \\
\text { nal } \\
(65 \%)\end{array}$} & \multirow{2}{*}{$\begin{array}{c}\text { Interna- } \\
\text { cional } \\
(29 \%)\end{array}$} & \multirow{2}{*}{$\begin{array}{c}\text { Ambos } \\
(6 \%)\end{array}$} \\
\hline & & & & \\
\hline $60 \%$ & $40 \%$ & - & - & - \\
\hline $35 \%$ & $0 \%$ & - & - & - \\
\hline $37 \%$ & $32 \%$ & - & - & - \\
\hline $25 \%$ & $18 \%$ & - & - & - \\
\hline $25 \%$ & $21 \%$ & - & - & - \\
\hline $12 \%$ & $12 \%$ & - & - & - \\
\hline $18 \%$ & $12 \%$ & - & - & - \\
\hline $26 \%$ & $26 \%$ & - & - & - \\
\hline $12 \%$ & $4 \%$ & - & - & - \\
\hline $35 \%$ & $23 \%$ & - & - & - \\
\hline $26 \%$ & $25 \%$ & - & - & - \\
\hline $44 \%$ & $56 \%$ & - & - & - \\
\hline $9 \%$ & $15 \%$ & $19 \%$ & $3 \%$ & - \\
\hline $40 \%$ & $41 \%$ & $50 \%$ & $25 \%$ & - \\
\hline $21 \%$ & $18 \%$ & $25 \%$ & $12 \%$ & - \\
\hline $21 \%$ & $9 \%$ & $13 \%$ & $13 \%$ & - \\
\hline $4 \%$ & $9 \%$ & $7 \%$ & $9 \%$ & - \\
\hline $9 \%$ & $3 \%$ & $9 \%$ & $4 \%$ & - \\
\hline $13 \%$ & $10 \%$ & $16 \%$ & $7 \%$ & - \\
\hline $4 \%$ & $3 \%$ & $7 \%$ & $1 \%$ & - \\
\hline
\end{tabular}




\begin{tabular}{|c|c|c|c|c|c|c|c|c|c|}
\hline Misiones & $0 \%$ & $0 \%$ & $0 \%$ & $0 \%$ & $0 \%$ & $0 \%$ & $0 \%$ & $0 \%$ & - \\
\hline Potenciar capacidades & $6 \%$ & $15 \%$ & $25 \%$ & $5 \%$ & $9 \%$ & $24 \%$ & $31 \%$ & $4 \%$ & - \\
\hline Facilitar Networking & $7 \%$ & $25 \%$ & $20 \%$ & $10 \%$ & $15 \%$ & $32 \%$ & $34 \%$ & $12 \%$ & - \\
\hline Creación- políticas públicas & $22 \%$ & $9 \%$ & $21 \%$ & $7 \%$ & $19 \%$ & $9 \%$ & $19 \%$ & $7 \%$ & - \\
\hline
\end{tabular}

Nota: a Las entidades de formación siempre tienen un alcance internacional, razón por la cual no aplica su clasificación - bSólo dinero. Fuente: Elaboración propia.

Para el caso del trabajo de campo en Colombia, en la entrevista se brindó la posibilidad a los organismos nacionales de clasificarse como oferentes tanto de servicios de formación como de servicios de apoyo. Así, de los 25 entrevistados, 12 se clasificaron como prestadores de sólo servicios de apoyo, 6 como prestadores de servicios sólo de formación y siete como prestadores de ambos (Figura 5).

\section{Organismos clasificados según el núcleo de las iniciativas ofrecidas}

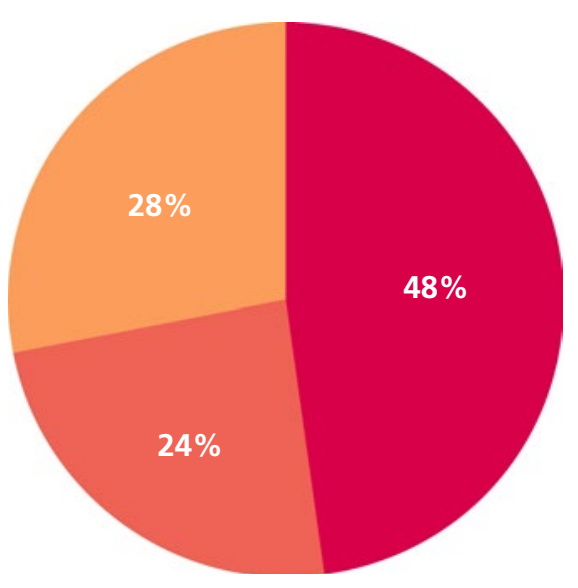

$\mathbf{4 8 \%}$ Solo apoyo

$\mathbf{2 4 \%}$ Solo formación

$\mathbf{2 8} \%$ Apoyo y formación

Figura 5

Organismos colombianos

clasificados según el núcleo de las

iniciativas ofrecidas.

Fuente: Elaboración propia. 


\title{
Tipo de organismos entrevistados
}

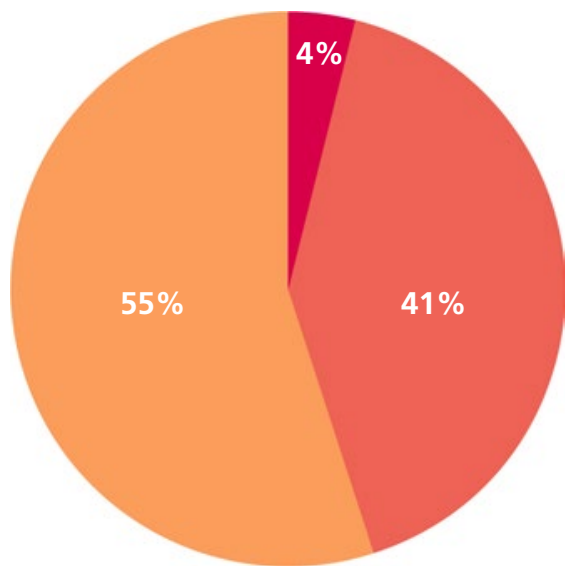
4\% Públicas
$\mathbf{4 1 \%}$ Privadas
$\mathbf{5 5 \%}$ Mixtas

\author{
Figura 6 \\ Tipos de organismos \\ entrevistados. \\ Fuente: Elaboración propia.
}

Otra información obtenida de la clasificación inicial corresponde al número de organismos privados, públicos y mixtos. Así, las 25 entrevistas corresponden a 22 organismos, nueve públicos, 12 privados y un mixto, estableciendo una relación de porcentajes (Figura 6).

El resultado de la relación del porcentaje de los esfuerzos dedicados por áreas de apoyo de los organismos en Colombia se muestra en la Figura 7.

\section{Porcentaje de esfuerzos concentrados por áreas}

\section{de apoyo en Colombia}

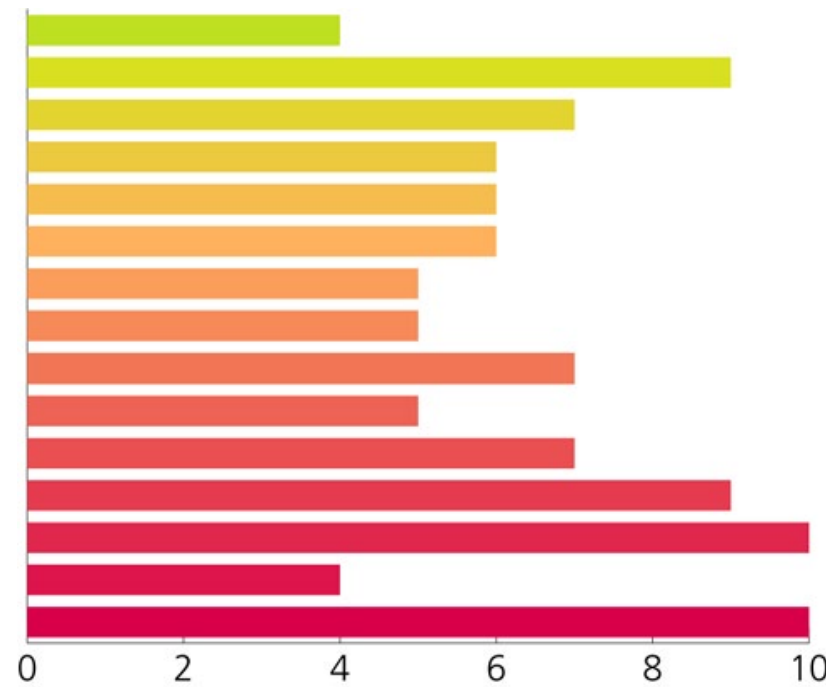

4\% Financiación (sólo dinero)

$\mathbf{9 \%}$ Ofrecer información propia/otros

$\mathbf{7 \%} \quad$ Hacer investigaciones del sector

$\mathbf{6 \%} \quad$ Realización de ruedas de negocio

6\% Realización de Ferias

$\mathbf{6 \%}$ Realización de muestras/Exposiciones

$\mathbf{5 \%} \quad$ Realización de Festivales

$\mathbf{5 \%}$ Realización de Misiones

7\% Realización de otros eventos

$\mathbf{5 \%} \quad$ Consultoría en estudios de mercado

$\mathbf{7 \%}$ Consultoría para diagnóstico

$\mathbf{9 \%} \quad$ Consultoría para potenciar capacidades

$10 \%$ Facilitando y/o propiciando elNetworking

4\% Incubación (apoyo recursos no \$)

$10 \%$ Creación de políticas públicas
Figura 7

Porcentaje de esfuerzos

concentrados por áreas

de apoyo en Colombia.

Fuente: Elaboración propia. 
La relación del porcentaje de los esfuerzos dedicados por áreas de temáticas de formación se muestra en la Figura 8.

\title{
Porcentaje de esfuerzos concentrados por áreas
}

\section{de formación en Colombia}

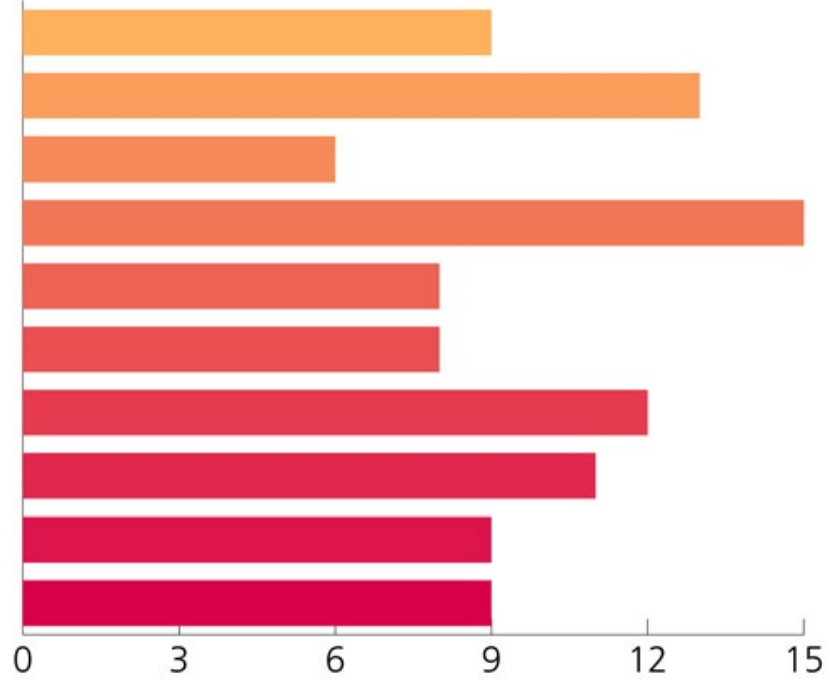

\author{
$\mathbf{9 \%} \quad$ Prácticas $=$ oficios y talentos \\ $13 \%$ Gestión empresarial \\ 6\% Investigación \\ 15\% Emprendimiento \\ $\mathbf{8 \%}$ Desarrollo de políticas públicas \\ $\mathbf{8 \%}$ Teoría y contexto \\ $12 \%$ Gestión comercial \\ 11\% Actualización \\ 9\% Formación para la sensibilidad \\ Porcentaje de esfuerzos \\ concentrados por \\ temáticas de formación \\ 9\% Gestión cultural \\ en Colombia. \\ Fuente: Elaboración propia.
}

Con los organismos colombianos se exploró adicionalmente la valoración que ellos hacen sobre la relevancia de los servicios de apoyo y formación ofrecidos, para tener un marco más amplio de interpretación sobre los resultados obtenidos, en función de la comparación con el estado de la oferta identificada en el mundo e Iberoamérica. Las Figuras 9 y 10 muestran cuántos de los entrevistados le asignan relevancia a cada área de esfuerzo específica en temas de apoyo y formación.

\section{Porcentaje de esfuerzos concentrados por áreas}

\section{de apoyo en Colombia}

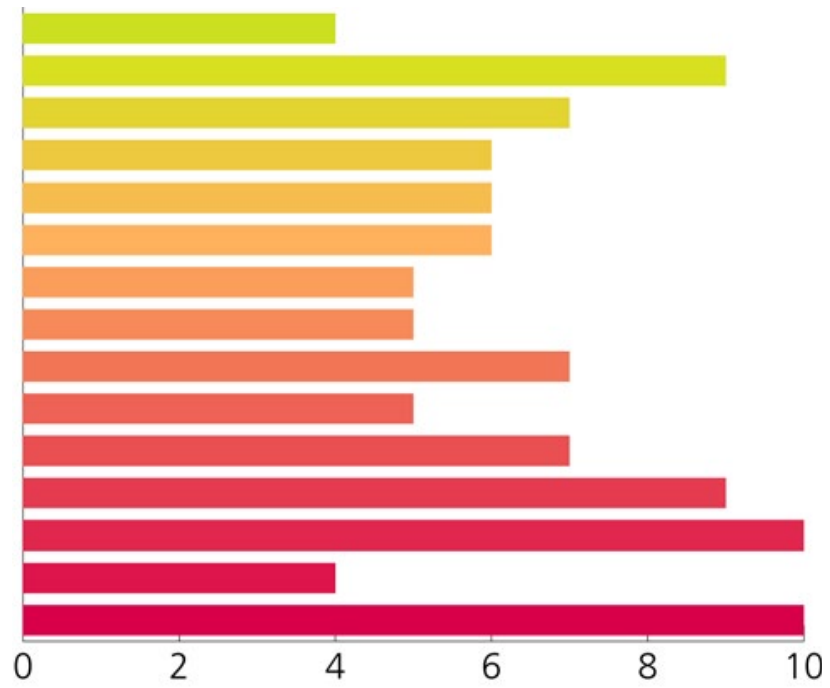

$\begin{array}{ll}\mathbf{4 \%} & \text { Financiación } \\ \mathbf{9 \%} & \text { Información } \\ \mathbf{7 \%} & \text { Investigación } \\ \mathbf{6 \%} & \text { Promoción de eventos } \\ \mathbf{6 \%} & \text { Promoción Ruedas de negocios } \\ \mathbf{6 \%} & \text { Promoción Ferias } \\ \mathbf{5 \%} & \text { Promoción Muestras/Exposiciones } \\ \mathbf{5 \%} & \text { Promoción Festivales } \\ \mathbf{7 \%} & \text { Promoción Misiones } \\ \mathbf{5 \%} & \text { Consultoría Estudios mercado } \\ \mathbf{7 \%} & \text { Consultoría Diagnóstico } \\ \mathbf{9 \%} & \text { Consultoría Potenciar capacidades } \\ \mathbf{1 0 \%} & \text { Networking } \\ \mathbf{4 \%} & \text { Incubación } \\ \mathbf{1 0 \%} & \text { Creación de políticas públicas }\end{array}$

Figura 9

Importancia asignada a los servicios de apoyo. Fuente: Elaboración propia. 


\section{Importancia asignada a los temas de formación}

Gestión cultural

Formación para la sensibilidad

Actualización

Gestión comercial

Teoría y contexto

Desarrollo de políticas públicas

Emprendimiento

Investigación

Gestión empresarial

Prácticas en oficios/talentos

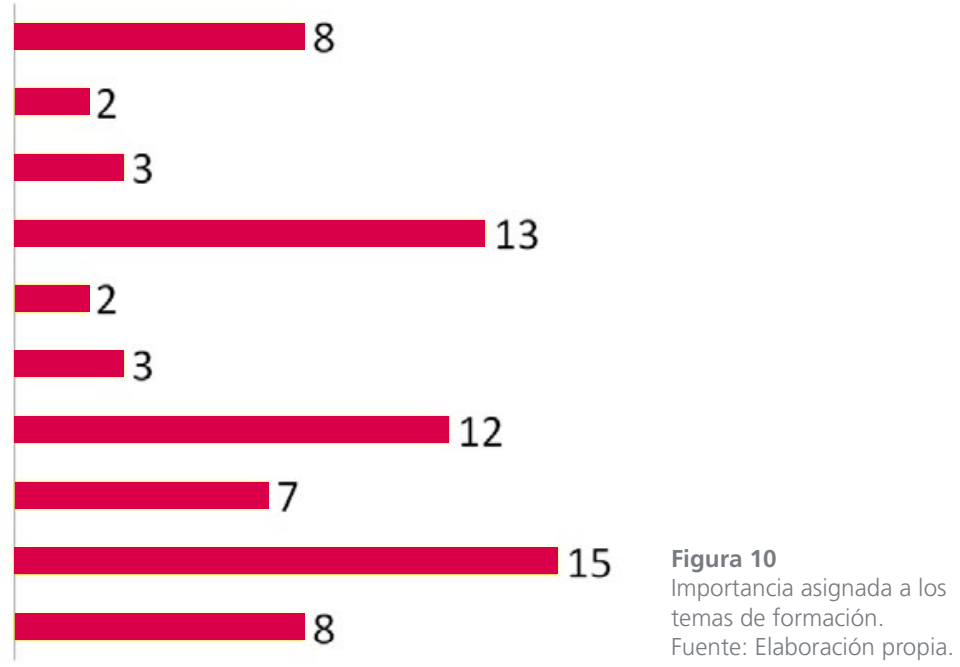

Adicionalmente, los entrevistados sugirieron otras temáticas adicionales que, en su opinión, deberían ser ofrecidas por medio de servicios de formación y apoyo. La consolidación de estas opiniones se muestra en la Tabla 9.

\section{Tabla 9. Recomendaciones adicionales para servicios de apoyo y formación}

\begin{tabular}{ll} 
Apoyo & Formación \\
\hline Desarrollo humano / aptitudes y actitudes. & Habilidades personales y creativas. \\
\hline $\begin{array}{l}\text { Fortalecer estructuras de empresa que protejan } \\
\text { la propiedad intelectual. }\end{array}$ & $\begin{array}{l}\text { Formalización y habilidades relacionadas } \\
\text { con la formalización. }\end{array}$ \\
\hline Capacitación presentación dossier. & Derechos de autor y propiedad intelectual. \\
\hline Actividades mercadológicas. & Fortalecimiento de la cadena de valor. \\
\hline & Desarrollo de producto y fortalecimiento. \\
\hline & Propiedad intelectual. \\
\hline
\end{tabular}

Fuente: Elaboración propia

Particularmente se resalta la importancia concedida por los entrevistados a los asuntos concernientes a la propiedad intelectual, así como a las actividades relacionadas con la formalización de los procesos empresariales y comerciales, y al fortalecimiento de la formación específica en los oficios artísticos. 
Al analizar la información recopilada en relación con el funcionamiento y componentes del ecosistema en Colombia, se identificó un listado de aquellos que actualmente hacen parte del ecosistema y un esquema de relaciones entre los organismos, según lo referenciado por los entrevistados. De este modo, se evidenciaron seis tipos de relaciones ecosistémicas entre los organismos denominadas así:

1 Relaciones de beneficio social: son relaciones establecidas entre organismos para poder prestar servicios de apoyo y formación a un grupo específico de la población.

2 Relaciones de beneficio financiero: son relaciones establecidas entre los organismos para conseguir respaldo de financiación para la puesta en marcha de las iniciativas.

3 Relaciones de beneficio formativo: son relaciones establecidas entre los organismos para prestar servicios de formación.

4 Relaciones de beneficio normativo: son relaciones establecidas entre los organismos para desarrollar procesos de generación de políticas públicas.

5 Relaciones de beneficio en servicios de apoyo: son relaciones establecidas entre los organismos para prestar servicios de apoyo.

6 Relaciones de beneficio de respaldo: son relaciones establecidas entre los organismos para establecer alianzas estratégicas que le den credibilidad a las iniciativas y programas ofrecidos.

La clasificación anterior emerge del ejercicio de análisis de los tipos de relación entre los organismos entrevistados y los actores por ellos referidos como aliados estratégicos para el cumplimiento de sus respectivos objetivos y el desarrollo de sus actividades. Así, los entrevistados refirieron en sus testimonios los nombres de otras instituciones con las que trabajan en el ecosistema y los objetivos que tienen para establecer dichas relaciones. Lo que se hizo evidente en los testimonios relacionados con este aspecto es que prima en los organismos el principio de asociación en la creación de relaciones entre los organismos del ecosistema, pero es más en función de una inercia de trabajo colaborativo, que por una predisposición explícita de orientar una asociación específica para robustecer alguno de los demás principios del ecosistema. Además, se hicieron evidentes 
muchas relaciones de interdependencia, poniendo en evidencia que los organismos son conscientes de la existencia articulada del ecosistema, aunque no hicieron evidente un orden puntual de causalidad para el establecimiento de tales relaciones. Finalmente, sobre los tipos de relaciones, también fue evidente que los organismos se asocian en función de conveniencia, experiencia previa con otros organismos o reputación de los mismos, y no en función de ampliar sus capacidades para adaptarse a futuras fluctuaciones del ecosistema, indicando que hay un trabajo por hacer en relación con el principio de flexibilidad.

\section{Discusión}

Esta investigación no pretende proponer una serie de prescripciones acerca de estrategias específicas para el desarrollo del emprendimiento cultural y creativo. Los resultados buscan ser un referente a partir de la noción de ecosistema, para orientar modelos o planteamientos específicos del quehacer de los organismos y componentes del mismo. En síntesis, la visión ecosistémica permite hacer interpretaciones para diagnosticar y desarrollar iniciativas de apoyo o formación para el emprendimiento cultural y creativo en diversos contextos.

La investigación permitió identificar las áreas de formación y apoyo en las cuales, en el mundo, Iberoamérica y Colombia, se están dedicando esfuerzos importantes, así como aquellas en las cuales la concentración de tales esfuerzos es más baja. Este análisis da indicios sobre las oportunidades de mejoramiento y fortalecimiento para este sector y ubica a Colombia dentro de un contexto global de formación y apoyo, permitiendo así a los organismos que ofrecen este tipo de iniciativas, evaluar y decidir sobre la pertinencia y concentración de su oferta. Con base en los hallazgos presentados, se presenta el análisis de la información en dos líneas principales: la precisión acerca de los resultados específicos más relevantes y la indicación de las oportunidades o implicaciones que tales resultados tienen para el crecimiento y fortalecimiento de los ecosistemas para el emprendimiento cultural y creativo. 
En relación con los resultados en torno a la formación que se muestran en la figura 3, es importante resaltar que los temas de gestión empresarial y gestión cultural en Iberoamérica presentan los porcentajes de esfuerzo del $21 \%$ y $23 \%$, respectivamente, los más altos, esto en contraste con el 15\% y $12 \%$, respectivamente, que se observa en el mundo en la Figura 1. Esto podría estar reflejando un interés hacia el fortalecimiento del sector en Iberoamérica, soportado en declaraciones como las que hace la UNCTAD (2008) sobre EC y su potencial para el desarrollo de las economías emergentes.

En contraste, se encuentra que la formación en investigación, desarrollo de políticas públicas y emprendimiento, con $6 \%, 5 \%$ y $4 \%$, respectivamente, son los más bajos de la Figura 3, para el caso de Iberoamérica. En el mundo, estos porcentajes son: teoría y contexto, desarrollo de política pública, investigación y actualización, con un $9 \%, 9 \%, 5 \%$ y $4 \%$, respectivamente, como se ve en la Figura 1. Para el caso del desarrollo de política pública, si bien es un porcentaje bajo en el mundo, la exploración bibliográfica mostró que no refleja una falta de política pública, sino, por el contrario, la existencia de fuertes y maduras políticas públicas, como es el caso del Reino Unido o Australia, donde no se crean nuevas líneas de formación para la generación de nuevos marcos políticos, lo que se encuentra son observatorios o centros de investigación que rastrean, mapean y alimentan la institucionalidad política existente y operativa. En Iberoamérica, sin embargo, este porcentaje bajo se puede interpretar, según los entrevistados y la exploración bibliográfica, como una falta en la definición e implementación de políticas públicas para la formalización y desarrollo del sector. Si bien en Colombia existen políticas culturales desde 1976 (Canelas y Bayaro, 2008) y leyes específicas sectoriales para la promoción y consolidación de los diferentes sectores que hacen parte de las ICC, los entrevistados señalaron que este aspecto está en una etapa aún inicial, y las implementaciones no encuentran aún sectores maduros de producción constante, excepto en años recientes, en industrias muy puntuales (música, cine, espectáculos públicos, entre otros). 
La formación en investigación y en emprendimiento para las ICC también son aspectos que aparecen con porcentajes bajos en ambos escenarios, lo cual señala que, al ser un sector con grandes expectativas de desarrollo, un mayor apoyo en la enseñanza de la investigación y el emprendimiento fortalecerán las ICC.

En conclusión, puede señalarse que la Figura 1 de esfuerzos en temáticas de formación en el mundo está balanceada en contraste con la Figura 3 de Iberoamérica. Esta investigación, y su enfoque desde la perspectiva del ecosistema, pueden aportar elementos para hacer que la fase de implementación sea balanceada en relación con los organismos que componen el ecosistema, en cuanto a sus necesidades y capacidades.

En lo relacionado con los servicios de apoyo, es posible observar que en ambos escenarios el esfuerzo por brindar información tiene el porcentaje más alto, con un 20\% (ver Figs. 2 y 4). Esto evidencia que los esfuerzos están concentrados en un servicio que requiere poca infraestructura y capacidades, y que puede ponerse fácilmente a disposición del sector por medio de las tecnologías de la información y la comunicación.

Se observa que el resto de los servicios se encuentran balanceados en ambos escenarios. Pero es necesario resaltar que existen dos porcentajes bajos, los relacionados con la promoción de misiones y los procesos de incubación, ambos con $0 \%$ y $1 \%$ respectivamente. Esto representa una oportunidad para el desarrollo del ecosistema en la medida que se consoliden estos dos aspectos para el fortalecimiento de las ICC.

Por su parte, en Colombia se encontró que la oferta en áreas de formación se encuentra balanceada con porcentajes que oscilan entre el $8 \%$ y el $15 \%$, como se observa en la gráfica 8 . Sin embargo, es necesario señalar que el porcentaje más elevado (15\%) corresponde a la formación en emprendimiento, pero a partir del trabajo de campo se percibe que se está entendiendo como formación en emprendimiento la formación en temas de gestión empresarial y comercial. 
No en vano ambos porcentajes se encuentran en valores similares (13\% y $12 \%$ ). En el país parece estar respondiendo a una necesidad de corto plazo para la formalización de las empresas del sector. Los entrevistados señalaron que las áreas más importantes de formación están en su orden: gestión empresarial, gestión comercial y emprendimiento. Analizando el discurso de los entrevistados, se percibe que no existe una distinción clara entre estos tres conceptos, y por lo tanto, entre las tres áreas de formación. Esto se constituye en otra oportunidad importante para el fortalecimiento del ecosistema en Colombia.

Por su parte, se encontró que la oferta en servicios de apoyo (ver Fig. 7), tiene mayor concentración en la facilitación del networking y en la creación de políticas públicas. Esto guarda similitud con la oferta de servicios de apoyo en el escenario mundial. Llama la atención que, si bien los servicios de incubación son los más bajos en el mundo e Iberoamérica, en Colombia presentan porcentajes más elevados. Sucede lo mismo con el porcentaje de promoción por medio de la realización de misiones. Esto puede indicar que los servicios de incubación en el mundo pueden tener debilidades en su modelo de operación en relación con todo el ecosistema; para el caso de las misiones, parecen ser un servicio que opera bajo la lógica de la intermitencia y la identificación de oportunidades específicas, más que como una estrategia con objetivos claramente definidos. Esta evidencia representa una vez más una oportunidad para el fortalecimiento del ecosistema en Colombia.

Los entrevistados señalaron que los servicios más importantes de apoyo (Fig. 9) son en su orden: financiación, creación de políticas públicas e investigación. La reflexión que se hace frente a esta evidencia es que es necesario conocer el sector en profundidad con el fin de dotarlo de unos marcos de acción legales y legítimos como parte de una política de Estado, que permitan a su vez atraer y generar un flujo constante y creciente de inversión y recursos financieros. 
En relación con estudios previos sobre la identificación y clasificación de los factores críticos de desempeño de las Empresas de Base Cultural y Creativa (Podestá \& Hernández, 2012), para establecer los aspectos que se deben considerar para el desarrollo del emprendimiento cultural y creativo, la investigación demostró que, si bien lo relacionado con los atributos humanos es un factor importante, no es determinante, es decir, existen factores mucho más relevantes para el cumplimiento de los objetivos de las ICC. Estos aspectos son los relacionados con la formalización de los procesos empresariales y aquellos que tienen que ver con la producción y materialización del acto creativo. Al compararlos con los resultados de la presente investigación, es posible afirmar que existe consistencia en el sentido en el que, por ejemplo, los organismos que ofrecen formación concentran sus esfuerzos en las áreas de gestión (empresarial y comercial) y emprendimiento, pero, además, afirman que son éstas las más importantes.

Por su parte, los organismos que ofrecen apoyo declaran que las ofertas de servicios de financiación y de política pública son las más importantes, lo cual guarda correspondencia con los factores críticos de desempeño referidos por Podestá y Hernández, en los cuales la financiación apareció como uno de los factores imprescindibles. Otros hallazgos de Podestá y Hernández también resaltaron la importancia de la formalización de los procesos de protección de los activos intelectuales y su explotación para las ICC, lo cual se corroboró en esta investigación (Podestá \& Hernández, 2012).

En la exploración bibliográfica sobre los servicios de apoyo ofrecidos, llamó la atención que los organismos de apoyo no declaran ofrecer apoyo en los procesos de protección a la propiedad intelectual, lo cual es similar en Colombia. Sin embargo, al pedirles a los entrevistados, su opinión sobre los aspectos que contribuirían a fortalecer el sector de las ICC, la protección de la propiedad intelectual aparece como un tema recurrente. Finalmente, se señala un aspecto adicional que se reveló en el trabajo de campo en lo relacionado con este tema. Como se puede observar en la Tabla 9, los entrevistados hicieron un fuerte énfasis en la 
necesidad que se ofrezcan servicios, tanto en formación como en apoyo, para el conocimiento y gestión de la propiedad intelectual en las ICC. Todo lo anterior se suma a críticas como las presentadas por Towse (2008), donde se señala que aún hay un camino por recorrer en esta línea.

\section{Futuras investigaciones}

Esta investigación llega a una primera identificación de ecosistemas para el emprendimiento cultural y creativo en Colombia y el mundo, mediante el mapeo de organismos, relaciones e intercambios que se hacen evidentes en ellos. Esta identificación permite hacer evidente las relaciones entre los organismos y la interdependencia entre ellos, al tiempo que se crea una guía de observación que sirva de base para mediciones y/o diagnósticos en relación con la sostenibilidad en el largo plazo para los ecosistemas. No obstante, la identificación de los ecosistemas es apenas el primer paso para comprender e intervenir la Economía Creativa en diferentes niveles de desarrollo y consolidación alrededor del mundo. Al establecer las relaciones entre los componentes de dicho ecosistema, es posible que los organismos involucrados en la evolución y sostenibilidad del ecosistema sean más pertinentes e identifiquen oportunidades de crecimiento.

Este estudio exploratorio sobre ecosistemas para el emprendimiento cultural y creativo recoge una evaluación preliminar que sienta las bases para estudios transversales y longitudinales posteriores, de forma que se rastreen el desarrollo, la maduración y el crecimiento de los ecosistemas y el fortalecimiento de las ICC, así como la pertinencia y la necesidad de acciones que las apoyen. Adicionalmente, de esta investigación se pueden derivar estudios que detallen los tipos de relaciones que se tejen entre los organismos que en este trabajo se enuncian, así como la incidencia de dichas relaciones en la dinámica y el desarrollo de los ecosistemas. Y en esta misma línea, se deja una base conceptual para explorar los principios de la naturaleza cíclica y la sostenibilidad de los ecosistemas, haciendo el seguimiento y el diagnóstico sobre la calidad y la efectividad de las iniciativas de formación y apoyo que se ponen en marcha. 
Queda la base entonces para expandir el alcance del análisis sobre estos dos principios en el tiempo, principios que por su longitudinalidad, no fueron cubiertos en este análisis.

Finalmente, el estudio es replicable allí donde se pretende rastrear la oferta de servicios de formación y apoyo a las ICC en los respectivos ecosistemas de Economía Creativa. Las herramientas de clasificación y categorización utilizadas en la investigación sirven de guía para consolidar un marco común que facilite la comparación entre diversos ecosistemas alrededor del mundo, construyéndose así un punto de partida para una reflexión de alcance mundial. Como bien lo indica la UNCTAD, las mediciones sobre la EC y las ICC deben ir evolucionando, y en ese mismo sentido, los diagnósticos sobre las características, el estado actual y la proyección de los ecosistemas también podrá ajustarse a las particularidades y estrategias de cada región, tomando como punto de partida los instrumentos construidos para esta investigación. 


\section{Referencias}

Bedoya, B. (2016). Los estudios económicos y las industrias culturales y creativas. International Journal of Management \& Social Studies, 1(1), 27-42.

Canelas, A., y Bayaro, R. (Orgs.) (2008). Politicas culturales en Iberoamérica. EDUFBA.

Capra, F. (1997). The Web of Life: A New Scientific Understanding of Living Systems. Harper Collins.

Cohen, B. (2006). Sustainable valley entrepreneurial ecosystems. Business Strategy and the Environment, 15(1), 1-14. https://doi. org/10.1002/bse.428

Del Rincón, D., Arnal, J., Latorre, A., \& Sans, A. (1995). Técnicas de investigación en ciencias sociales. Dykinson.

Feld, B. (2012). Startup communities: Building an entrepreneurial ecosystem in your city. John Wiley \& Sons Inc.

Isenberg, D. J. (2010). The Big Idea: How to Start an Entrepreneurial Revolution. Harvard Business Review, 88(6), 40-50.

Mack, E., \& Mayer, H. (2016). The evolutionary dynamics of entrepreneurial ecosystems. Urban Studies, 53(10), 2118-2133. https://doi.org/10.1177/0042098015586547

Mason, C., \& Brown, R. (2014). Entrepreneurial ecosystems and growth oriented entrepreneurship. OECD. https://www.oecd.org/ cfe/leed/entrepreneurial-ecosystems.pdf

Podestá, P., \& Hernández, M. (2012). Empresas de base cultural y creativa: identificación y clasificación de sus factores críticos de desempeño. TEC Empresarial, 6(2), 17-27. https://doi.org/10.18845/te.v6i2.526

Towse, R. (2008). Why has Cultural Economics Ignored Copyright? Journal of Cultural Economics, 32, 243-259. https://doi.org/10.1007/s10824-008-9080-0
UNCTAD. (2008). Creative Economy Report 2008. UNCTAD. https://unctad.org/en/Docs/ ditc20082cer_en.pdf

UNCTAD. (14 de Diciembre de 2010). Creative Economy Report 2010. UNCTAD. https://unctad.org/en/Docs/ditctab20103_en.pdf

UNCTAD. (14 de Noviembre de 2013). Creative Economy Report 2013. UNCTAD. http://www.unesco.org/culture/pdf/creativeeconomy-report-2013.pdf

Vargas, J. I. (2012). La entrevista en la investigación cualitativa: nuevas tendencias y retos. Revista Calidad en la Educación Superior, 3(1), 119-139. http://biblioteca.icap.ac.cr/BLIVI/ COLECCION_UNPAN/BOL_DICIEMBRE_2013_69/ UNED/2012/investigacion_cualitativa.pdf 